PROCEEDINGS OF THE

AMERICAN MATHEMATICAL SOCIETY

Volume 131, Number 5, Pages 1471-1479

S 0002-9939(02)06661-3

Article electronically published on September 20, 2002

\title{
AN APPROACH TO THE SPECTRUM STRUCTURE OF DIRAC OPERATORS BY THE LOCAL-COMPACTNESS METHOD
}

\author{
TADASHI IKUTA AND KAZUHISA SHIMA \\ (Communicated by N. Tomczak-Jaegermann)
}

\begin{abstract}
The purpose of this paper is to investigate the spectra of the Dirac operator $H=H_{0}+V=-i c \alpha \cdot \nabla+\beta m c^{2}+V$. The local compactness of $H$ is shown under some assumption on $V$. This method enables us to prove that if $|V(x)-a \beta| \rightarrow 0$ as $|x| \rightarrow \infty$, then $\sigma_{\text {ess }}(H)=\left(-\infty,-m c^{2}-a\right] \cup\left[m c^{2}+a, \infty\right)$ and to give a significant sufficient condition that $H^{+}$or $H^{-}$has a purely discrete spectrum.
\end{abstract}

\section{INTRODUCTION}

Recently the behavior of spectra of the Dirac operator has been investigated by several authors. In such investigation the concept of local compactness of an operator in $L^{2}\left(\mathbb{R}^{l}\right)$ plays an important role and is well matched with the Kato perturbation theory [9]. This concept was first emphasized by Enss [6], adopted by Thaller [14 and incorporated in the work of Hislop and Sigal [7]. It also has a close relation to the Zhislin spectrum [17], which allows us to derive the behavior of essential spectra in a simpler way. This spectrum is also useful in investigating under what condition the Dirac operator has a purely discrete spectrum.

In $\S 2$, the concept of local compactness is defined and the basic properties of a locally compact operator are newly summarized in three theorems. Subsequently, the Zhislin sequence is introduced as a special case of the Weyl sequence. It leads to the fundamental theorem which states the relation between the Zhislin spectrum and the essential spectrum of the operator concerned.

In $\S 3$, the free Dirac operator $H_{0}$ is briefly outlined and its spectrum is definitely described. The local compactness of $H_{0}$ is shown by using that of the Laplacian $-\Delta$.

In $\S 4$, our study is to research the spectra of a perturbed Dirac operator $H=$ $H_{0}+V$ with a $4 \times 4$ Hermitian matrix potential $V$. It is shown that if $V$ is $H_{0^{-}}$ bounded with a bound less than 1 and $\lim _{|x| \rightarrow \infty}|V(x)-a \beta|=0$, then the essential spectrum is shifted away from the origin, that is, $\sigma_{\text {ess }}(H)=\left(-\infty,-m c^{2}-a\right] \cup$ $\left[m c^{2}+a, \infty\right)$. In case $V$ is a diagonal matrix $\left(V_{1}, V_{2}, V_{3}, V_{4}\right)$ composed of continuous

Received by the editors November 29, 2000 and, in revised form, March 29, 2001 and December $11,2001$.

1991 Mathematics Subject Classification. Primary 34L05, 34L40.

Key words and phrases. Locally compact operator, Dirac operator, essential spectrum, discrete spectrum. 
functions, it is proved that if some components of $V$ diverge to $+\infty$ and the other to $-\infty$ as $|x| \rightarrow \infty$, then each $H^{ \pm}\left(:=H_{0}^{ \pm}+V^{ \pm}\right)$has a purely discrete spectrum. The three theorems in $\S 4$ are our main ones in this paper.

\section{LOCALly COMPACT OPERATORS IN $L^{2}\left(\mathbb{R}^{l}\right)$}

For any bounded measurable subset $\mathcal{K}$ of $\mathbb{R}^{l}$ let $\chi_{\mathcal{K}}$ be the characteristic function of $\mathcal{K}$. We will use the same notation as the multiplication operator in $L^{2}\left(\mathbb{R}^{l}\right)$. This is an orthogonal projection operator. With the help of the operator $\chi_{\mathcal{K}}$ we introduce the concept of local compactness of an operator in $L^{2}\left(\mathbb{R}^{l}\right)$.

Definition 2.1 (locally compact operator). Let $A$ be a closed operator in $L^{2}\left(\mathbb{R}^{l}\right)$ with $\rho(A) \neq \emptyset$. Then $A$ is said to be locally compact if for each bounded measurable set $\mathcal{K}$, the product of $\chi_{\mathcal{K}}$ and the resolvent of $A, \chi_{\mathcal{K}}(A-z)^{-1}$, is compact for some (and hence all) $z \in \rho(A)(\rho(A)$ denotes the resolvent set of $A$ ).

It follows immediately that if $A$ is locally compact, then $A+k I$ is also locally compact for all $k \in \mathbb{C}$.

Example 2.2. The Laplacian $-\Delta$ is locally compact on $H^{2}\left(\mathbb{R}^{l}\right)$ with $l=1,2,3$ (see e.g. [13, Theorem 4.1]).

Here for all $s \geq 0$, the Sobolev space of order $s, H^{s}\left(\mathbb{R}^{l}\right)$, is the set of function $f \in L^{2}\left(\mathbb{R}^{l}\right)$ whose Fourier transform $\widehat{f}$ obeys

$$
\|f\|_{H^{s}}^{2}:=\int_{\mathbb{R}^{l}}|\widehat{f}(\xi)|^{2}\left(1+\xi^{2}\right)^{s} d \xi<\infty .
$$

For $s \in \mathbb{N}, f \in H^{s}\left(\mathbb{R}^{l}\right)$ if and only if, for all multi-indices $\alpha$ with $|\alpha| \leq s$, the distributional derivative

$$
D^{\alpha} f(x)=\frac{\partial^{|\alpha|} f}{\partial x_{1}^{\alpha_{1}} \cdots \partial x_{l}^{\alpha_{l}}}(x)
$$

lies in $L^{2}\left(\mathbb{R}^{l}\right)$, and that $\sum_{|\alpha| \leq s}\left\|D^{\alpha} f\right\|^{2}$ and $\|f\|_{H^{s}}^{2}$ are equivalent norms.

The following three theorems are fundamental in proving the local compactness of the Dirac operator.

Theorem 2.3. If $A$ is a self-adjoint and locally compact operator in $L^{2}\left(\mathbb{R}^{l}\right)$ and $B$ is a symmetric and $A$-bounded operator with $A$-bound less than 1 , then $A+B$ is self-adjoint and locally compact in $D(A+B)=D(A)$.

Proof. The self-adjointness of $A+B$ is an immediate result of the Kato-Rellich theorem. Our aim is to show the local compactness of $A+B$. We obtain

$$
\chi_{\mathcal{K}}(A+B-z)^{-1}=\chi_{\mathcal{K}}(A-z)^{-1}-\chi_{\mathcal{K}}(A-z)^{-1} B(A+B-z)^{-1}
$$

by the second resolvent identity, for all $z \in \rho(A+B) \cap \rho(A)$. Our assumption implies that there are two positive constants $a, b$ with $a<1$ for which we have

$$
\begin{aligned}
\|B u\| & \leq a\|A u\|+b\|u\| \\
& \leq a\|(A+B) u\|+a\|B u\|+b\|u\|
\end{aligned}
$$

for any $u \in D(A)$. Hence we get

$$
\|B u\| \leq \frac{a}{1-a}\|(A+B) u\|+\frac{b}{1-a}\|u\| .
$$


This means that $B$ is $(A+B)$-bounded. Since $B(A+B-z)^{-1}$ is bounded, we can conclude that $A+B$ is locally compact.

Theorem 2.4. Let $A$ be a self-adjoint operator in $L^{2}\left(\mathbb{R}^{l}\right)$. If $A^{2}$ is locally compact, then $A$ is also locally compact.

Proof. Let $\mathcal{K}$ be a bounded measurable subset of $\mathbb{R}^{l}$ and put $B:=(i+A)^{-1} \chi_{\mathcal{K}}$. Then both $B$ and $B^{*}$ are bounded and we have

$$
B^{*} B=\chi_{\mathcal{K}}\left(1+A^{2}\right)^{-1} \chi_{\mathcal{K}} .
$$

Hence the local-compactness of $A^{2}$ implies that $B^{*} B$ is compact. Now let $\left\{u_{n}\right\}$ be a sequence in $L^{2}\left(\mathbb{R}^{l}\right)$ which converges weakly to 0 . Clearly, $\left\{\left\|u_{n}\right\|\right\}$ is bounded and $\left\{B^{*} B u_{n}\right\}$ converges strongly to 0 . At once the inequality

$$
\left\|B u_{n}\right\|^{2} \leq\left\|u_{n}\right\|\left\|B^{*} B u_{n}\right\|
$$

leads to the strong convergence of $\left\{B u_{n}\right\}$ to 0 . Hence $B$ is compact and so is $B^{*}$. This means that $A$ is locally compact.

For any self-adjoint operator $A$ in $L^{2}\left(\mathbb{R}^{l}\right)$ we put

$$
A^{ \pm}:=\frac{1}{2}(A \pm|A|)
$$

where $|A|$ is the absolute value of $A$. Then $A^{+}$is non-negative and $A^{-}$is nonpositive in $L^{2}\left(\mathbb{R}^{l}\right)$.

Theorem 2.5. Let $A$ be a self-adjoint operator in $L^{2}\left(\mathbb{R}^{l}\right)$. If $A$ is locally compact, then $A^{ \pm}$are also locally compact.

Proof. Let $A_{\varepsilon}^{ \pm}=\frac{1}{2}(A \pm \varepsilon|A|)(0<\varepsilon<1)$. Then $A_{\varepsilon}^{ \pm}$are locally compact in $L^{2}\left(\mathbb{R}^{l}\right)$ because $\varepsilon|A|$ is $A$-bounded with $A$-bound 0 . By the second resolvent identity we obtain

$$
\left\|\chi_{\mathcal{K}}\left(A_{\varepsilon}^{+}-i\right)^{-1}-\chi_{\mathcal{K}}\left(A^{+}-i\right)^{-1}\right\| \leq \frac{|\varepsilon-1|}{2}\left\|\left(A_{\varepsilon}^{+}-i\right)^{-1}|A|\left(A^{+}-i\right)^{-1}\right\| .
$$

Hence $\chi_{\mathcal{K}}\left(A_{\varepsilon}^{+}-i\right)^{-1}$ converges to $\chi_{\mathcal{K}}\left(A^{+}-i\right)^{-1}$ in the sense of operator norm as $\varepsilon$ tends to 1 . Therefore $\chi_{\mathcal{K}}\left(A^{+}-i\right)^{-1}$ is compact, that is, $A^{+}$is locally compact.

The local compactness of $A^{-}$is similarly proved.

Now, to analyze the essential spectra of a locally compact and self-adjoint operator, it is important to introduce the Weyl sequence and the Zhislin sequence. By $B_{r}(0)$ we denote the closed ball with center 0 and radius $r$.

Definition 2.6 (Weyl sequence [15]). Let $A$ be a closed operator in $L^{2}\left(\mathbb{R}^{l}\right)$. A sequence $\left\{u_{n}\right\}$ in $D(A)$ is called a Weyl sequence for $A$ and $\lambda \in \mathbb{C}$ if

$$
\left\|u_{n}\right\|=1, \quad \text { w- } \lim _{n \rightarrow \infty} u_{n}=0 \quad \text { and } \quad \text { s- } \lim _{n \rightarrow \infty}(A-\lambda) u_{n}=0 .
$$

Definition 2.7 (Zhislin sequence [17]). Let $A$ be a closed operator in $L^{2}\left(\mathbb{R}^{l}\right)$. A sequence $\left\{u_{n}\right\}$ in $D(A)$ is called a Zhislin sequence for $A$ and $\lambda \in \mathbb{C}$ if

$$
\left\|u_{n}\right\|=1, \quad \operatorname{supp}\left(u_{n}\right) \subset \mathbb{R}^{l} \backslash B_{n}(0) \quad \text { and } \quad \text { s- } \lim _{n \rightarrow \infty}(A-\lambda) u_{n}=0 .
$$


It is clear that a Zhislin sequence for a closed operator $A$ and $\lambda \in \mathbb{C}$ is a Weyl sequence for the same $A$ and $\lambda$. It should be remarked that if there exists a Zhislin sequence for a self-adjoint operator $A$ and $\lambda \in \mathbb{C}$, then $\lambda$ is an essential spectrum of $A$ (the precise definition of essential spectrum is given in Definition 2.9).

Definition 2.8 (Zhislin spectrum and Weyl spectrum). Let $A$ be a closed operator in $L^{2}\left(\mathbb{R}^{l}\right)$. Then the set of all $\lambda \in \mathbb{C}$ such that there exists a Weyl sequence for $A$ and $\lambda$ is called the Weyl spectrum, which is denoted by $W(A)$. Similarly, the Zhislin spectrum is the set of all $\lambda \in \mathbb{C}$ such that there exists a Zhislin sequence for $A$ and $\lambda$, which is denoted by $Z(A)$. Then the relation $Z(A) \subset W(A) \subset \sigma(A)$ holds $(\sigma(A)$ denotes the spectrum of $A)$.

Definition 2.9. Let $A$ be a self-adjoint operator. The set composed of all accumulation points of $\sigma(A)$ and of all isolated eigenvalues of $A$ with infinite multiplicity is called the essential spectrum of $A$ and denoted by $\sigma_{\text {ess }}(A)$.

The complement of $\sigma_{\text {ess }}(A)$ in $\sigma(A), \sigma(A) \backslash \sigma_{\text {ess }}(A)$, is called the discrete spectrum of $A$ and denoted by $\sigma_{\mathrm{d}}(A)$. If $\sigma(A)=\sigma_{\mathrm{d}}(A)$, we say that $A$ has a purely discrete spectrum.

The following theorem states that $\sigma_{\mathrm{ess}}(A)=Z(A)$ under some condition. Since $Z(A)$ is easier to determine than $\sigma_{\text {ess }}(A)$, the theorem is useful to investigate $\sigma_{\text {ess }}(A)$.

Theorem 2.10. Let $A$ be a locally compact operator in $L^{2}\left(\mathbb{R}^{l}\right)$ and suppose that for some $z \in \rho(A)$, A also satisfies

$$
\left\|\left[A, \psi_{n}\right](A-z)^{-1}\right\| \rightarrow 0 \quad(n \rightarrow \infty),
$$

where $\psi_{n}(x)=\psi\left(n^{-1} x\right)$ for some $\psi \in C_{0}^{\infty}\left(\mathbb{R}^{l}\right), \operatorname{supp}(\psi) \subset B_{2}(0), \psi \geq 0$ and $\left.\psi\right|_{B_{1}(0)}=1$. Here the commutator of $A$ and $\psi_{n}$ is defined by $\left[A, \psi_{n}\right] g:=A\left(\psi_{n} g\right)-$ $\psi_{n}(A g)$ for all $g \in D(A)$. Then $\sigma_{\mathrm{ess}}(A) \subset Z(A)$. In addition, if $A$ is self-adjoint, then $\sigma_{\mathrm{ess}}(A)=Z(A)$ (For the proof, see [7]).

Remark 2.11. This theorem will be used in $\S 4$ in a slightly extended form. The direct sum of $\overbrace{L^{2}\left(\mathbb{R}^{l}\right) \oplus \cdots \oplus L^{2}\left(\mathbb{R}^{l}\right)}^{r \text { times }}$ is abbreviated as $\left[L^{2}\left(\mathbb{R}^{l}\right)\right]^{r}$. If $A$ is a locally compact operator in $\left[L^{2}\left(\mathbb{R}^{l}\right)\right]^{r}$, use the following expression:

$$
\left\|\left[A, \psi_{n} I_{r}\right](A-z)^{-1}\right\| \rightarrow 0 \quad(n \rightarrow \infty)
$$

instead of (2.4).

\section{Free Dirac operator}

The free Dirac operator $H_{0}$ in the Hilbert space $\mathfrak{H}=\left[L^{2}\left(\mathbb{R}^{3}\right)\right]^{4} \cong L^{2}\left(\mathbb{R}^{3}\right) \otimes \mathbb{C}^{4}$ is given by

$$
H_{0}=-i c \boldsymbol{\alpha} \cdot \nabla+\beta m c^{2}
$$

where $\alpha_{j}(j=1,2,3), \beta=\alpha_{0}$ are $4 \times 4$ constant Hermitian matrices satisfying the anti-commutator relations

$$
\alpha_{j} \alpha_{k}+\alpha_{k} \alpha_{j}=2 \delta_{j k} I_{4}
$$


( $I_{k}$ denotes a $k \times k$ identity matrix) and $m, c$ are positive constants. In this paper, we put

$$
\begin{aligned}
& \boldsymbol{\alpha}=\left(\alpha_{1}, \alpha_{2}, \alpha_{3}\right), \quad \alpha_{j}=\left(\begin{array}{cc}
\mathbf{0} & \sigma_{j} \\
\sigma_{j} & \mathbf{0}
\end{array}\right), \quad \beta=\left(\begin{array}{cc}
I_{2} & \mathbf{0} \\
\mathbf{0} & -I_{2}
\end{array}\right), \\
& \sigma_{1}=\left(\begin{array}{ll}
0 & 1 \\
1 & 0
\end{array}\right), \quad \sigma_{2}=\left(\begin{array}{cc}
0 & -i \\
i & 0
\end{array}\right), \quad \sigma_{3}=\left(\begin{array}{cc}
1 & 0 \\
0 & -1
\end{array}\right) .
\end{aligned}
$$

The inner product in $\mathfrak{H}$ is given by

$$
\langle f, g\rangle=\sum_{j=1}^{4} \int_{\mathbb{R}^{3}} \overline{f_{j}(x)} g_{j}(x) d x
$$

for all 4-component column vectors $f={ }^{t}\left(f_{1}, f_{2}, f_{3}, f_{4}\right), g={ }^{t}\left(g_{1}, g_{2}, g_{3}, g_{4}\right) \in \mathfrak{H}$.

The free Dirac operator has the following properties (see e.g. [14, 15]):

(1) $H_{0}$ is essentially self-adjoint on the dense domain $C_{0}^{\infty}\left(\mathbb{R}^{3}\right)^{4}$ and self-adjoint on $H^{1}\left(\mathbb{R}^{3}\right)^{4}$.

(2) $\sigma\left(H_{0}\right)=\sigma_{\text {ess }}\left(H_{0}\right)=\left(-\infty,-m c^{2}\right] \cup\left[m c^{2}, \infty\right)$.

We are now in a position to prove the local compactness of $H_{0}$ by using that of the Laplacian $-\Delta$.

Theorem 3.1. The free Dirac operator $H_{0}$ is locally compact on $H^{1}\left(\mathbb{R}^{3}\right)^{4}$.

Proof. Note that the domain of $H_{0}^{2}$ is equal to $H^{2}\left(\mathbb{R}^{3}\right)^{4}$. Indeed, it is clear that $H^{2}\left(\mathbb{R}^{3}\right)^{4} \subset D\left(H_{0}^{2}\right)$ and the inverse inclusion relation follows from the fact that if $f, \partial_{j} f \in H^{s}\left(\mathbb{R}^{l}\right), j=1, \ldots, l$, then $f \in H^{s+1}\left(\mathbb{R}^{l}\right)$ and

$$
\|f\|_{H^{s+1}}^{2} \leq\|f\|_{H^{s}}^{2}+\sum_{j=1}^{l}\left\|\partial_{j} f\right\|_{H^{s}}^{2} .
$$

The above statement shows that $H_{0}^{2}=c^{2}\left(-\Delta+m^{2} c^{2}\right)$ is locally compact on $H^{2}\left(\mathbb{R}^{3}\right)^{4}$. Therefore, $H_{0}$ is also locally compact by Theorem 2.4.

\section{Dirac operators With Matrix Potential}

In this section, we consider a perturbed Hamiltonian $H=H_{0}+V$, where $V$ is a $4 \times 4$ Hermitian matrix and investigate the behavior of the spectrum of $H$. For this purpose, it is convenient to introduce the absolute value of $V$ as follows:

$$
|V(x)|:=\sqrt{\sum_{i, j=1}^{4}\left|V_{i j}(x)\right|^{2}} .
$$

Theorem 4.1. Let $V$ be a multiplication operator in $\mathfrak{H}$ with a Hermitian $4 \times 4$ matrix and $H_{0}$-bounded with $H_{0}$-bound less than 1 . If

$$
\lim _{|x| \rightarrow \infty}|V(x)-a \beta|=0
$$

for some $a \geq 0$, then $H$ is self-adjoint and locally compact on $D\left(H_{0}\right)=\left(H^{1}\left(\mathbb{R}^{3}\right)\right)^{4}$. Furthermore, we obtain

$$
\sigma_{\mathrm{ess}}(H)=\left(-\infty,-m c^{2}-a\right] \cup\left[m c^{2}+a, \infty\right) .
$$


Proof. It follows from Theorem 2.3 and Theorem 3.1 that $H$ is self-adjoint and locally compact. With the help of Theorem 2.10 we can prove the final assertion $\sigma_{\text {ess }}(H)=\sigma_{\text {ess }}(T)$, where $T=H_{0}+a \beta$.

Note that for all $f \in D(H)$, we have

$$
\left[H, \psi_{n} I_{4}\right] f=\left[H_{0}, \psi_{n} I_{4}\right] f=c\left[\boldsymbol{\alpha} \cdot \boldsymbol{p}, \psi_{n} I_{4}\right] f=c \sum_{j=1}^{4} f_{j} \boldsymbol{\alpha} \cdot\left(\boldsymbol{p} \varphi_{n} \boldsymbol{e}_{j}\right),
$$

where $\boldsymbol{p}=-i \nabla$ and $\boldsymbol{e}_{j}$ is the 4-component column vector with 1 at the $j$ th place and zero otherwise. This implies $Z(H)=\sigma_{\text {ess }}(H)$ and $Z(T)=\sigma_{\text {ess }}(T)$ by (2.5).

We first show that $\sigma_{\text {ess }}(H) \subset \sigma_{\text {ess }}(T)$. Let $\lambda \in \sigma_{\text {ess }}(H)$ and $\left\{u_{n}\right\}$ be a Zhislin sequence for $H$ and $\lambda$. We remark that $u_{n} \in D(T)$ from $H_{0}$-boundedness of $V$. Since $\operatorname{supp}\left(u_{n}\right) \subset\left(\mathbb{R}^{3} \backslash B_{n}(0)\right)^{4}$, we have

$$
\left\|(V-a \beta) u_{n}\right\| \leq \sup \left\{|V(x)-a \beta| ; x \in \mathbb{R}^{3} \backslash B_{n}(0)\right\} \rightarrow 0 \quad(n \rightarrow \infty)
$$

by (4.2) and hence we get

$$
\left\|(T-\lambda) u_{n}\right\| \leq\left\|(H-\lambda) u_{n}\right\|+\left\|(V-a \beta) u_{n}\right\| \rightarrow 0 \quad(n \rightarrow \infty) .
$$

Therefore, $u_{n}$ is also a Zhislin sequence for $T$ and $\lambda$, that is, $\lambda \in Z(T)=\sigma_{\text {ess }}(T)$.

To prove the converse, let $\lambda \in \sigma_{\text {ess }}(T)=Z(T)$. Then there exists a Zhislin sequence $\left\{u_{n}\right\}$ for $T$ and $\lambda$. Again, the vanishing of $V-a \beta$ at infinity implies that $\left\|(V-a \beta) u_{n}\right\| \rightarrow 0$ as $n \rightarrow \infty$, so we obtain

$$
\left\|(H-\lambda) u_{n}\right\| \leq\left\|(T-\lambda) u_{n}\right\|+\left\|(V-a \beta) u_{n}\right\| \rightarrow 0 .
$$

This proves that $\left\{u_{n}\right\}$ is a Zhislin sequence for $H$ and $\lambda$. Hence $\lambda \in Z(H)=$ $\sigma_{\text {ess }}(H)$.

Subsequently we must investigate what condition upon $V$ makes $H$ possess a purely discrete spectrum, but this is a difficult problem in comparison with the Schrödinger operator, $-\Delta+V$. Here we shall study a sufficient condition that $H^{ \pm}:=H_{0}^{ \pm}+V^{ \pm}$has a purely discrete spectrum. To do so, it is necessary to check the local compactness of $H^{ \pm}$.

Theorem 4.2. Let $V$ be a multiplication operator in $\mathfrak{H}$ with a Hermitian $4 \times 4$ matrix and let $V^{ \pm}$and $H_{0}^{ \pm}$be as in (2.1). Suppose each component of $V$ is a continuous function on $\mathbb{R}^{3}$. Then $H^{ \pm}:=H_{0}^{ \pm}+V^{ \pm}$are essentially self-adjoint and the closed extensions of them are locally compact.

Proof. Let $S^{ \pm}=\frac{1}{2} H_{0}+V^{ \pm}$. The essential self-adjointness of $S^{ \pm}$follows immediately from Theorem 5.6 of Jörgens [8]. Since $\frac{1}{2}\left|H_{0}\right|$ is $S^{+}$-bounded with $S^{+}$-bound $1, H^{+}$is essentially self-adjoint. We denote the closed extension of $H^{+}$by the same symbol. Since $H^{+} \geq 0$ and $H_{0}^{+} \geq 0$, both $\left(H^{+}+1\right)^{1 / 2}$ and $\left(H_{0}^{+}+1\right)^{1 / 2}$ are well-defined. Obviously we have

$$
\left\|\left(H_{0}^{+}+1\right)^{1 / 2} u\right\|^{2}=\left\langle u,\left(H_{0}^{+}+1\right) u\right\rangle \leq\left\langle u,\left(H^{+}+1\right) u\right\rangle=\left\|\left(H^{+}+1\right)^{1 / 2} u\right\|^{2}
$$

for all $u \in D\left(\left(H^{+}+1\right)^{1 / 2}\right)$. Consequently, $\left(H_{0}^{+}+1\right)^{1 / 2}\left(H^{+}+1\right)^{-1 / 2}$ is bounded. On the other hand, Theorem[2.5 shows that $H_{0}^{+}$is locally compact and hence $\left(H_{0}^{+}\right)^{1 / 2}$ is. These imply that

$$
\chi_{\mathcal{K}}\left(H^{+}+1\right)^{-1 / 2}=\chi_{\mathcal{K}}\left(H_{0}^{+}+1\right)^{-1 / 2}\left(H_{0}^{+}+1\right)^{1 / 2}\left(H^{+}+1\right)^{-1 / 2}
$$


is compact. Hence $H^{+}$is locally compact, since

$$
\chi_{\mathcal{K}}\left(H^{+}+1\right)^{-1}=\chi_{\mathcal{K}}\left(H^{+}+1\right)^{-1 / 2}\left(H^{+}+1\right)^{-1 / 2}
$$

is compact.

The local compactness of $H^{-}$is similarly proved, since $-H^{-}$is non-negative on $\mathfrak{H}$.

Remark 4.3. Since $H$ is not always locally compact, we cannot invoke Theorem 2.5 for $H$.

Theorem 4.4. Let $V$ be a diagonal Hermitian $4 \times 4$ matrix,

$$
V=\left(\begin{array}{cccc}
V_{1} & & & 0 \\
& V_{2} & & \\
0 & & V_{3} & \\
& & & V_{4}
\end{array}\right)
$$

Suppose that each $V_{j}$ is a continuous function on $\mathbb{R}^{3}$ and has a definite sign outside $B_{r}(0)$ for a sufficiently large $r$.

(1) If each non-negative component of $V$ diverges to $\infty$ as $|x| \rightarrow \infty$, then $\mathrm{H}^{+}$ has a purely discrete spectrum.

(2) If each non-positive component of $V$ diverges to $-\infty$ as $|x| \rightarrow \infty$, then $\mathrm{H}^{-}$ has a purely discrete spectrum.

Proof. We prove a special case when $V_{1}, V_{2} \geq 0$ and $V_{3}, V_{4} \leq 0$ for sufficiently large $|x|$. Then we have

$$
V^{+}=\left(\begin{array}{cccc}
V_{1} & & & 0 \\
& V_{2} & & \\
0 & & 0 & \\
0 & & & 0
\end{array}\right), \quad V^{-}=\left(\begin{array}{cccc}
0 & & & \\
& 0 & & \\
& & & V_{4}
\end{array}\right) .
$$

(1) Suppose $\sigma_{\text {ess }}\left(H^{+}\right) \neq \emptyset$. Theorem 4.2 shows that $H^{+}$is locally compact in $\mathfrak{H}$. Let $\lambda \in \sigma_{\text {ess }}\left(H^{+}\right)=Z\left(H^{+}\right)$. Then there exists a Zhislin sequence $\left\{u_{n}\right\}$ for $H^{+}$ and $\lambda$. Subsequently, for a sufficiently large $n$, we obtain the estimate

$$
\begin{aligned}
\left\|\left(\lambda-H^{+}\right) u_{n}\right\| & \geq\left|\left\langle u_{n},\left(\lambda-H^{+}\right) u_{n}\right\rangle\right| \geq\left\langle u_{n}, V^{+} u_{n}\right\rangle-|\lambda| \\
& \geq C_{n}\left(1-\int_{\mathbb{R}^{3} \backslash B_{n}(0)}\left(\left|u_{3}^{(n)}(x)\right|^{2}+\left|u_{4}^{(n)}(x)\right|^{2}\right) d x\right)-|\lambda|,
\end{aligned}
$$

where

$$
C_{n}=\min \left(\inf _{x \in \mathbb{R}^{3} \backslash B_{n}(0)} V_{1}(x), \inf _{x \in \mathbb{R}^{3} \backslash B_{n}(0)} V_{2}(x)\right) .
$$

The left most side of (4.5) converges to 0 as $n \rightarrow \infty$, while $C_{n} \rightarrow \infty$ and the integrated part converges to 0 by our assumption. This is a contradiction. Hence we must have $\sigma_{\text {ess }}\left(H^{+}\right)=\emptyset$, that is, $\sigma\left(H^{+}\right)=\sigma_{\mathrm{d}}\left(H^{+}\right)$.

(2) Use the inequality

$$
\left\|\left(\lambda-H^{-}\right) v_{n}\right\| \geq D_{n}\left(1-\int_{\mathbb{R}^{3} \backslash B_{n}(0)}\left(\left|u_{1}^{(n)}(x)\right|^{2}+\left|u_{2}^{(n)}(x)\right|^{2}\right) d x\right)-|\lambda|,
$$


where

$$
D_{n}=\min \left(\inf _{x \in \mathbb{R}^{3} \backslash B_{n}(0)}\left(-V_{3}(x)\right), \inf _{x \in \mathbb{R}^{3} \backslash B_{n}(0)}\left(-V_{4}(x)\right)\right),
$$

with the Zhislin sequence $\left\{v_{n}\right\}$ for $H^{-}$and $\lambda$.

The other cases are similarly proved.

Corollary 4.5. In case the four components are non-negative (respectively nonpositive), we have $\sigma\left(H^{+}\right)=\sigma_{\mathrm{d}}\left(H^{+}\right)$and $\sigma\left(H^{-}\right)=\left(-\infty,-m c^{2}\right] \cup\{0\}$ (respectively $\sigma\left(H^{+}\right)=\{0\} \cup\left[m c^{2}, \infty\right)$ and $\left.\sigma\left(H^{-}\right)=\sigma_{\mathrm{d}}\left(H^{-}\right)\right)$.

Proof. We only have to prove the second equation in the former case. Put $\varphi^{ \pm}(x):=$ $\frac{1}{2}(x \pm|x|)$. Then we get

$$
\sigma\left(H_{0}^{-}\right)=\overline{\varphi^{-}\left(\sigma\left(H_{0}\right)\right)}=\left(-\infty,-m c^{2}\right] \cup\{0\}
$$

by virtue of the spectral mapping theorem (3], Theorem 8.11). Hence we obtain $\sigma\left(H^{-}\right)=\sigma\left(H_{0}^{-}\right)=\left(-\infty,-m c^{2}\right] \cup\{0\}$.

\section{Discussion}

In Theorem 4.4 the relation $H=H^{+}+H^{-}$holds, but the spectrum relation $\sigma(H)=\sigma\left(H^{+}\right) \cup \sigma\left(H^{-}\right)$is not always valid. Even in the special case which appears in the beginning of the proof, we are not ready to demonstrate the proposition $\sigma(H)=\sigma_{\mathrm{d}}\left(H^{+}\right) \cup \sigma_{\mathrm{d}}\left(H^{-}\right)$at this stage. Theorem 4.4 states that each $H^{ \pm}$has a purely discrete spectrum in the respective case concerned. Our forthcoming task is to research under what condition upon $V$ the total Hamiltonian $H$ has a purely discrete spectrum. It remains as an important problem, furthermore, to extend Theorem 4.4 to the case when $V$ has off-diagonal parts.

As an application of Corollary 4.5, we consider the case when $V(x)=f(x) \beta+$ $g(x) I_{4}$, where $f$ and $g$ are real-valued continuous functions on $\mathbb{R}^{3}$.

(1) If $g \geq f \geq 0$ and $\lim _{|x| \rightarrow \infty} g(x)=+\infty$, then we have $\sigma\left(H^{+}\right)=\sigma_{\mathrm{d}}\left(H^{+}\right)$and $\sigma\left(H^{-}\right)=\left(-\infty,-m c^{2}\right] \cup\{0\}$.

(2) If $g \leq f \leq 0$ and $\lim _{|x| \rightarrow \infty} g(x)=-\infty$, then we have $\sigma\left(H^{+}\right)=\{0\} \cup\left[m c^{2},+\infty\right)$ and $\sigma\left(H^{-}\right)=\sigma_{\mathrm{d}}\left(H^{-}\right)$.

This is similar to Yamada's result [16], but we cannot assert which result has a wider application.

Theorem 3.1 was referred to by Thaller [14. We have proved it concisely by reducing it to the local compactness of $-\Delta$.

\section{ACKNOWLEDGEMENTS}

We express our sincere gratitude to Professor K. Furutani and Professor N. Otuski of Department of Mathematics for their stimulating encouragement and valuable discussions.

\section{REFERENCES}

1. M. Arai, On essential Self-Adjointness of Dirac Operators, RIMS Kokyuroku 242 (1975), $10-21$.

2. M. Arai and O. Yamada, Essential Self-adjointness and Invariance of the Essential Spectrum for Dirac Operators, Publ. RIMS 18 (1982), 973-985. 
3. J. B. Conway, A Course in Functional Analysis, 2nd ed., Springer-Verlag, New York, Tokyo, 1990. MR 91e:46001

4. H. L. Cycon, R. G. Froese, W. Kirsch, and B. Simon, Schrödinger Operators, with Applications to Quantum Mechanics and Global Geometry, Springer-Verlag, Berlin, 1987. MR 88g:35003

5. P. Deift, W. Hunziker, B. Simon, and E. Vock, Pointwise Bounds on Eigenfunctions and Wave Packets in N-Body Quantum Systems $N$, Commun. Math. Phys. 64 (1978), 1-34. MR 80k:81016

6. V. Enss, Geometrical Methods in Spectral and Scattering Theory of Schrödinger Operators, in Rigorous Atomic and Molecular Physics edited by G. Velo and A. S. Wightman, Plenum, New York, 1981, 7-69.

7. P. D. Hislop and I. M. Sigal, Introduction to Spectral Theory, With Application to Schrödinger Operators, Applied Mathematical Sciences, vol. 113, Springer, New York, 1996.

8. K. Jörgens, Perturbations of the Dirac Operator. Conference on the theory of ordinary and partial differential equations, Lecture Note in Mathematics, vol. 280, Springer-Verlag, Berlin, Heidelberg, New York, Tokyo, 1980. MR 54:8047

9. T. Kato, Perturbation Theory for Linear Operators, Corrected Printing of 2nd ed., SpringerVerlag, Berlin, 1980. MR 96a:47025

10. S. Nakamura, Lectures on Schrödinger operators, Lectures in Mathematical Sciences, vol. 6, Graduate School of Mathematical Sciences, University of Tokyo, 1994

11. P. A. Perry, Scattering Theory by the Enss Method, Mathematical Reports, vol. 1, Harwood, New York, 1983. MR 85k:35181

12. M. Reed and B. Simon, Methods of Modern Mathematical Physics, vol. I-IN, Academic Press, New York, 1972-1979. MR 58:12429a; MR 58:12429b MR 80m:81085; MR 58:12429c

13. B. Simon, Trace Ideals and Their Applications, London Mathematical Society Lecture Note Series, vol. 35, Cambridge University Press, 1979. MR 80k:47048

14. B. Thaller, The Dirac Equation, Texts and Monographs in Physics, Springer-Verlag, BerlinHeidelberg-New York, 1992. MR 94k:81056

15. J. Weidmann, Linear Operators in Hilbert Spaces, English ed., Springer-Verlag, Berlin, 1980. MR 81e: 47001

16. O. Yamada, On the Spectrum of Dirac Operators with the Unbounded Potential at Infinity, Hokkaido Math. J. 26 (1997), 439-449. MR 98e:35122

17. G. Zhislin, Discussion of the Spectrum of the Schrödinger Operator for Systems of Several Particles, Tr. Mosk. Mat. Obs. 9 (1960), 81-128.

Department of Mathematics, Faculty of Science and Technology, Science University of Tokyo, Noda, ChiBa 278-8510, JAPAN

E-mail address: ikuta_tadashi@ma.noda.tus.ac.jp

Department of Mathematics, Faculty of Science and Technology, Science University of TOKYO, NodA, Chiba 278-8510, JAPAN

E-mail address: shima@rs.noda.tus.ac.jp 